\title{
Effects of information-dependent vaccination behavior on coronavirus outbreak: insights from a SIRI model
}

\section{Bruno Buonomo ${ }^{1}$}

Received: 22 February 2020 / Accepted: 22 March 2020 / Published online: 9 April 2020

(c) Università degli Studi di Napoli "Federico II" 2020

\begin{abstract}
A mathematical model is proposed to assess the effects of a vaccine on the time evolution of a coronavirus outbreak. The model has the basic structure of SIRI compartments (susceptible-infectious-recovered-infectious) and is implemented by taking into account of the behavioral changes of individuals in response to the available information on the status of the disease in the community. We found that the cumulative incidence may be significantly reduced when the information coverage is high enough and/or the information delay is short, especially when the reinfection rate is high enough to sustain the presence of the disease in the community. This analysis is inspired by the ongoing outbreak of a respiratory illness caused by the novel coronavirus COVID-19.
\end{abstract}

Keywords Epidemic model · Coronavirus · Reinfection · Vaccine $\cdot$ Information

Mathematics Subject Classification 92D30 - 34C60

\section{Introduction}

On 7 January 2020, China announced the outbreak of a respiratory illness caused by a novel coronavirus which did not match any other known virus. The coronavirus was temporarily named 2019-nCoV and finally COVID-19 [32]. The outbreak was first detected in Wuhan City, Hubei Province, and then expanded in the rest of China and other countries [31]. Coronaviruses are a large family of viruses which can affect many different species of animals, like cattle, cats, and bats. Rarely, animal coron-

This paper is dedicated to Prof. G. Toscani and Prof. M. Sugiyama on the occasion of their 70th birthday.

\footnotetext{
$\bowtie$ Bruno Buonomo

buonomo@unina.it

1 Department of Mathematics and Applications, University of Naples Federico II, via Cintia, 80126 Naples, Italy
} 
aviruses can infect people and then spreading from person-to-person. However, this may happen with serious consequences: well known cases are that of Severe acute respiratory syndrome (SARS) which killed 813 people worldwide during 2002-2003 outbreak [29], and the more recent case of Middle East respiratory syndrome coronavirus (MERS), where a total of 2494 confirmed cases including 858 associated deaths were reported, the majority from Saudi Arabia (at the end of November 2019, [30]). Therefore, coronavirus may represent a serious public health threat.

The emergency related to the novel outbreak in China is still ongoing at time of writing this article and it is unclear how the situation worldwide will unfold. The news released by media create great concern and behavioral changes can be observed in the everyday life of individuals, even in Europe where at the moment only few cases have been reported. For example, the fear of coronavirus has driven rapidly to sold out of protective face masks in pharmacies in Italy long before the first case in the country was reported [1].

A specific aspects of diseases caused by coronavirus is that humans can be reinfected with respiratory coronaviruses throughout life [19]. The duration of immunity for SARS, for example, was estimated to be greater than 3 years [34]. Moreover, investigations on human coronavirus with infected volunteers has shown that even though the immune system react after the infection (serum-specific immunoglobulin and IgC antibody levels peak 12-14 days after infection) at one year following experimental infection there is only partial protection against re-infection with the homologous strain [9].

Predictions or insight concerning the time-evolution of epidemics, especially when a new emerging infectious disease is under investigation, can be obtained by using mathematical models. In Mathematical Epidemiology, a large amount of literature is devoted to the use of the so called compartmental epidemic models, where the individuals of the community affected by the infectious disease are divided in mutually exclusive groups (the compartments) according to their status with respect to the disease $[3,4,10,21,24]$. Compartmental epidemic models are providing to be the first mathematical approach for estimating the epidemiological parameter values of COVID-19 in its early stage and for anticipating future trends [2,11,28].

When the disease under interest confer permanent immunity from reinfection after being recovered, the celebrated SIR model (susceptible-infectious-recovered) and its many variants are most often adopted. However, where reinfection cannot be neglected the SIRS model (susceptible-infectious-recovered, and again susceptible) and its variants may be used, under the assumption that infection does not change the host susceptibility $[3,4,10,21,24]$.

Since the disease of our interest has both reinfection and partial immunity after infection, we consider as starting point the so-called SIRI model (susceptibleinfectious-recovered-infectious) which takes into account of both these features (see [25] and the references contained therein for further information on SIRI model). When the epidemic process may be decoupled from the longer time-scale demographic dynamics, i. e. when birth and natural death terms may be neglected, one gets a simpler model with an interesting property. In fact, according to the values of three relevant parameters (the transmission rate, the recovery rate and the reinfection rate), the model exhibits three different dynamics [18,20]: (i) no epidemic will occur, in the 
sense that the fraction of infectious will decrease from the initial value to zero; (ii) an epidemic outbreak occurs, in the sense that the fraction of infectious will initially increase till a maximum value is reached and then it decreases to zero; (iii) an epidemic outbreak occurs and the disease will permanently remain within the population.

At time of writing this paper, scholars are racing to make a vaccine for the novel COVID-19 coronavirus available. As of February 12, 2020, it was announced that 'The first vaccine could be ready in 18 months' [32]. Therefore, it becomes an intriguing problem to qualitatively assess how the administration of a vaccine could affect the outbreak, taking into account of the behavioral changes of individuals in response to the information available on the status of the disease in the community. This is the main aim of this paper.

The scenario depicted here is that of a community where a relatively small quantity of infectious is present at time of delivering the vaccine. The vaccination is assumed to be fully voluntary and the choice to get vaccinated or not is assumed to depend in part on the available information and rumors concerning the spread of the disease in the community.

The behavioral change of individuals is introduced by employing the method of information-dependent models $[14,15,33]$ which is based on the introduction of a suitable information index. Such an approach has been applied to general infectious diseases $[8,14,15,23,33]$ as well as specific ones, including childhood diseases like measles, mumps and rubella [14,33] and is currently under development (for very recent papers see [5,22,35]). Therefore, another goal of this manuscript is to provide an application of the information index to a simple model containing relevant features of a coronavirus disease. Specifically, we use epidemiological parameter values based on early estimation of novel coronavirus COVID-19 [28].

The rest of the paper is organized as follows: in Sect. 2 we introduce the basic SIRI model and recall its main properties. In Sect. 3 we implement the SIRI model by introducing the information-dependent vaccination. The epidemic and the reinfection thresholds are discussed in Sect. 4. Section 5 is devoted to numerical investigations: the effects of the information parameters on the time evolution of the outbreak are discussed. Conclusions and future perspective are given in Sect. 6.

\section{The SIRI model}

Since the disease of our interest has both reinfection and partial immunity after infection, we first consider the SIRI model, which is given by the following nonlinear ordinary differential equations (the upper dot denotes the time derivative) [18]:

$$
\begin{aligned}
& \dot{S}=\mu(1-S)-\beta S I \\
& \dot{I}=\beta S I+\sigma \beta R I-(\gamma+\mu) I \\
& \dot{R}=-\sigma \beta R I+\gamma I-\mu R .
\end{aligned}
$$

Here $S, I$ and $R$ denote, respectively, the fractions of susceptible, infectious (and also infected) and recovered individuals, at a time $t$ (the dependence on $t$ is omitted); 
$\beta$ is the transmission rate; $\gamma$ is the recovery rate; $\mu$ is the birth/death rate; $\sigma \in(0,1)$ is the reduction in susceptibility due to previous infection.

Model (1) assumes that the time-scale under consideration is such that demographic dynamics must be considered.

However, epidemics caused by coronavirus often occurs quickly enough to neglect the demographic processes (as in the case of SARS in 2002-2003). When the epidemic process is decoupled from demography, i.e. when $\mu=0$, one obviously gets the reduced model:

$$
\begin{aligned}
& \dot{S}=-\beta S I \\
& \dot{I}=\beta S I+\sigma \beta R I-\gamma I \\
& \dot{R}=-\sigma \beta R I+\gamma I .
\end{aligned}
$$

This very simple model has interesting properties. Indeed, introduce the basic reproduction number $R_{0}=\beta / \gamma$. It has been shown that the solutions have the following behavior [20]:

If $R_{0} \leq 1$, then no epidemic will occur, in the sense that the state variable $I(t)$ denoting the fraction of infectious will decrease from the initial value to zero;

If $R_{0} \in(1,1 / \sigma)$, then an epidemic outbreak will follow, in the sense that the state variable $I(t)$ will initially increase till a maximum value is reached and then it decreases to zero;

If $R_{0}>1 / \sigma$, then an epidemic outbreak will follow and the disease will permanently remain within the population, in the sense that the state variable $I(t)$ will approach (after a possibly non monotone transient) an endemic equilibrium $E$, given by:

$$
E=(\bar{S}, \bar{I}, \bar{R})
$$

where:

$$
\bar{S}=0 ; \quad \bar{I}=1-\frac{1}{\sigma R_{0}} ; \quad \bar{R}=\frac{1}{\sigma R_{0}} .
$$

The equilibrium $E$ is globally asymptotically stable [20] and it is interesting to note that, since the demography has been neglected, the disease will persist in the population due to the reservoir of partially susceptible individuals in the compartment $R$.

From a mathematical point of view, the threshold $R_{0}=R_{0 \sigma}$, where $R_{0 \sigma}=1 / \sigma$, is a bifurcation value for model (2). This does not happen for model (1). In fact, when demography is included in the model, the endemic equilibrium exists for $R_{0}>1$, where $R_{0}=\beta /(\mu+\gamma)$ and therefore both below and above the reinfection threshold.

Model (2) (as well as (1)) is a simple model which is able to describe the timeevolution of the epidemic spread on a short time-scale. However, it does not takes into account of possible control measure. The simplest one to consider is vaccination. We consider the scenario where the vaccination is assumed to be fully voluntary. In order to emphasize the role of reinfection, we assume that only susceptible individuals (i.e. individuals that did not experience the infection) consider this protective option. When 
the vaccine is perfect (i.e. it is an ideal vaccine which confer 100 percent life-long immunity) one gets the following model:

$$
\begin{aligned}
& \dot{S}=-\beta S I-\varphi_{0} S \\
& \dot{I}=\beta S I+\sigma \beta R I-\gamma I \\
& \dot{R}=-\sigma \beta R I+\gamma I \\
& \dot{V}=\varphi_{0} S .
\end{aligned}
$$

where $V$ denotes the fraction of vaccinated individuals and $\varphi_{0}$ is the vaccination rate.

In the next section we will modify the SIRI model (4) to assess how an hypothetical vaccine could control the outbreak, taking into account of the behavioral changes of individuals produced by the information available on the status of the disease in the community.

\section{The information-dependent model}

We modify the SIRI model by employing the idea of the information-dependent epidemic models $[23,33]$. We assume that the vaccination is fully voluntary and information-dependent, in the sense that the choice to get vaccinated or not depends on the available information and rumors concerning the spread of the disease in the community.

The information is mathematically represented by an information index $M(t)$, which summarizes the information about the current and past values of the disease and is given by the following distributed delay [12-14,16]:

$$
M(t)=\int_{-\infty}^{t} \tilde{g}(S(\tau), I(\tau), V(\tau)) K(t-\tau) d \tau,
$$

Here, the function $\tilde{g}$ describes the information that individuals consider to be relevant for making their choice to vaccinate or not to vaccinate. It is often assumed that $\tilde{g}$ depends only on prevalence $[5,12,14,16]$

$$
\tilde{g}=\left\{\begin{array}{ll}
0 & \text { if } \quad t<0 \\
g(I) & \text { if } \quad t \geq 0
\end{array},\right.
$$

where $g$ is a continuous, differentiable, increasing function such that $g(0)=0$. In particular, we assume that:

$$
g(I)=k I \text {. }
$$

In (6) the parameter $k$ is the information coverage and may be seen as a 'summary' of two opposite phenomena, the disease under-reporting and the level of media coverage of the status of the disease, which tends to amplify the social alarm. The range of variability of $k$ may be restricted to the interval $(0,1)$ (see [6]). 
Fig. 1 The

information-dependent vaccination rate (8) as function of the information index $M$. The parameter values are given in Table 1

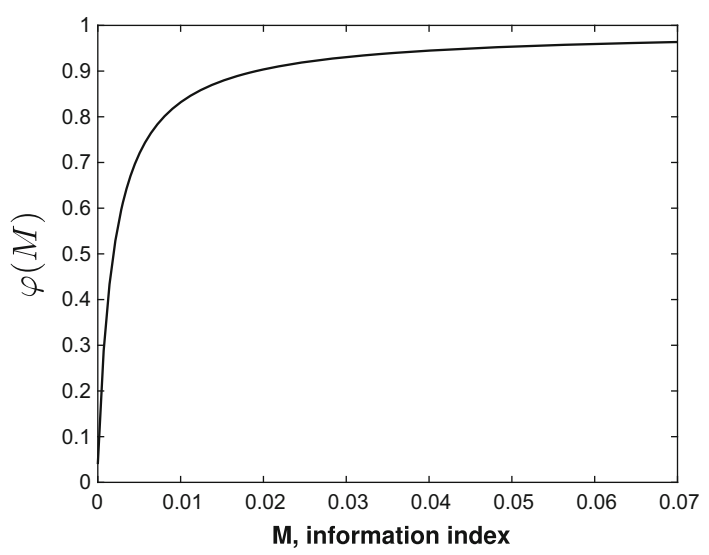

The delay kernel $K(t)$ in (5) is a positive function such that $\int_{0}^{+\infty} K(t) d t=1$ and represents the weight given to past history of the disease. We assume that the kernel is given by the first element $\operatorname{Erl}_{1, a}(t)$ of the Erlangian family, called weak kernel or exponentially fading memory. This means that the maximum weight is assigned to the current information and the delay is centered at the average $1 / a$. Therefore, the parameter $a$ takes the meaning of inverse of the average time delay of the collected information on the disease. With this choice, by applying the linear chain trick [26], the dynamics of $M$ is ruled by the equation:

$$
\dot{M}=a(k I-M) \text {. }
$$

We couple this equation with model (4). The coupling is realized through the following information-dependent vaccination rate:

$$
\varphi(M)=\varphi_{0}+\varphi_{1}(M)
$$

where the constant $\varphi_{0} \in(0,1)$ represents the fraction of the population that chooses to get vaccinate regardless of rumors and information about the status of the disease in the population, and $\varphi_{1}(M(t))$ represents the fraction of the population whose vaccination choice is influenced by the information.

Generally speaking, we require that $\varphi_{1}(0)=0$ and $\varphi_{1}$ is a continuous, differentiable and increasing function. However, as done in [5,14], we take:

$$
\varphi_{1}(M)=\left(1-\varphi_{0}-\varepsilon\right) \frac{D M}{1+D M},
$$

where $\varepsilon>0$. This parametrization leads to an overall coverage of $1-\varepsilon$ (asymptotically for $M \rightarrow \infty$ ). Here we take $\varepsilon=0.01$, which means a roof of $99 \%$ in vaccine uptakes under circumstances of high perceived risk. We also take $D=500$ [14]. Note that this choice of parameter values implies that a $96.4 \%$ vaccination coverage is obtained in correspondence of an information index $M=0.07$ (see Fig. 1). 
Table 1 Description and baseline values of the parameters of model (9)

\begin{tabular}{llll}
\hline Parameter & Meaning & Baseline value & Reference \\
\hline$\beta$ & Transmission rate & $1.07 \mathrm{ind}^{-1} \mathrm{day}^{-1}$ & {$[28]$} \\
$\gamma$ & Recovery rate & $0.278 \mathrm{day}^{-1}$ & {$[28]$} \\
$\sigma$ & Rel. susceptibility of recovered indiv. & - & Varying \\
$\psi$ & Rel. susceptibility of vaccinated indiv. & 0.15 & Guessed \\
$\varphi_{0}$ & Information-independent vaccinaton rate & $0.04 \mathrm{day}^{-1}$ & {$[5]$} \\
$k$ & Information coverage & $(0.2,1)$ & Guessed \\
$1-\epsilon$ & Max vaccin. coverage due to information & 0.99 & {$[5]$} \\
$D$ & Michaelis-Menten parameter & 500 & {$[14]$} \\
$T$ & Average time delay of information & $(0,120)$ day & Guessed \\
$a$ & Inverse average time delay of infor. $(1 / T)$ & $(0.00833, \infty)$ & Guessed \\
\hline
\end{tabular}

Finally we assume that the vaccine is not perfect, which is a more realistic hypothesis, so that the vaccinated individuals may be infected but with a reduced susceptibility $\psi$.

The SIRI epidemic model with information-dependent vaccination that we consider is therefore given by

$$
\left\{\begin{array}{l}
\dot{S}=-\beta S I-\varphi(M) S \\
\dot{I}=\beta S I+\sigma \beta R I+\psi \beta V I-\gamma I \\
\dot{R}=-\sigma \beta R I+\gamma I \\
\dot{V}=-\psi \beta V I+\varphi(M) S \\
\dot{M}=a(k I-M)
\end{array}\right.
$$

The meaning of the state variables, the parameters and their baseline values are given in Table 1.

Note that

$$
\frac{d}{d t}(S+I+R+V)=0,
$$

therefore assuming that $S(0)+I(0)+R(0)+V(0)=1$ we can substitute $S=$ $1-I-R-V$ in (9) to get:

$$
\left\{\begin{array}{l}
\dot{I}=\beta(1-I-R-V) I+\sigma \beta R I+\psi \beta V I-\gamma I \\
\dot{R}=-\sigma \beta R I+\gamma I \\
\dot{V}=\varphi(M)(1-I-R-V)-\psi \beta V I \\
\dot{M}=a(k I-M)
\end{array}\right.
$$




\section{The epidemic and the reinfection thresholds}

\subsection{The epidemic threshold}

Let us introduce the quantity

$$
\mathcal{P}_{0}=\frac{\beta}{\gamma},
$$

which is the basic reproduction number of model (2) [20]. From the second equation of (9) it easily follows that

$$
\dot{I}=\gamma I[\mathcal{P}(S, R, V)-1]
$$

where:

$$
\mathcal{P}(S, R, V)=\mathcal{P}_{0} S+\sigma \mathcal{P}_{0} R+\psi \mathcal{P}_{0} V
$$

It immediately follows that, if $I(0)>0$, then:

$$
\mathcal{P}(S(0), R(0), V(0))>1 \Longleftrightarrow I^{\prime}(0)>0,
$$

and

$$
\mathcal{P}(S(0), R(0), V(0))<1 \Longleftrightarrow I^{\prime}(0)<0 .
$$

Assuming that $I(0)>0$ and $R(0)=V(0)=0$ (and therefore $S(0)<1$ ) it follows that:

If $\mathcal{P}_{0}<1 / S(0)$, then the epidemic curve initially decays. If $\mathcal{P}_{0}>1 / S(0)$ the epidemic takes place since the infectious curve initially grows.

\subsection{The reinfection threshold}

From the first equation in (9) it can be seen that at equilibrium it must be $\tilde{S}=0$. Therefore, all the possible equilibria are susceptible-free. Since the solutions are clearly bounded, this means that for large time any individual who was initially susceptible has experienced the disease or has been vaccinated. Looking for equilibria in the form $\tilde{E}=(\tilde{I}, \tilde{R}, \tilde{V}, \tilde{M})$, from (10) we get:

$$
\left\{\begin{array}{l}
\beta(1-\tilde{I}-\tilde{R}-\tilde{V}) \tilde{I}+\sigma \beta \tilde{R} \tilde{I}+\psi \beta \tilde{V} \tilde{I}-\gamma \tilde{I}=0 \\
-\sigma \beta \tilde{R} \tilde{I}+\gamma \tilde{I}=0 \\
\varphi(\tilde{M})(1-\tilde{I}-\tilde{R}-\tilde{V})-\psi \beta \tilde{V} \tilde{I}=0 \\
a(k \tilde{I}-\tilde{M})=0
\end{array}\right.
$$


Disease-free equilibria: If $\tilde{I}=0$. It can be easily seen from (12) that

$$
\varphi_{0}(1-\tilde{R}-\tilde{V})=0
$$

Therefore there are infinitely many disease-free equilibria of the form

$$
E_{0}=\left(0, R_{0}, V_{0}, 0\right),
$$

where $R_{0}+V_{0}=1$.

Endemic equilibrium: We begin by looking for equilibria such that

$$
\tilde{I} \neq 0 ; \quad \tilde{S}=1-\tilde{I}-\tilde{R}-\tilde{V}=0 .
$$

This implies that:

$$
\left\{\begin{array}{l}
\sigma \beta \tilde{R} \tilde{I}+\psi \beta \tilde{V} \tilde{I}-\gamma \tilde{I}=0 \\
-\sigma \beta \tilde{R} \tilde{I}+\gamma \tilde{I}=0 \\
\psi \beta \tilde{V} \tilde{I}=0 \\
k \tilde{I}=\tilde{M}
\end{array}\right.
$$

Therefore: $\tilde{V}=0$ and

$$
\tilde{R}=\frac{\gamma}{\sigma \beta}
$$

and from (13):

$$
\tilde{I}=1-\tilde{R}
$$

It follows that an unique susceptibles-free endemic equilibrium exists, which is given by:

$$
E_{1}=\left(I_{1}, R_{1}, 0, M_{1}\right)
$$

where

$$
I_{1}=1-\frac{1}{\sigma \mathcal{P}_{0}} ; \quad R_{1}=\frac{1}{\sigma \mathcal{P}_{0}} ; \quad M_{1}=k I_{1},
$$

which exists only if

$$
\mathcal{P}_{0}>\frac{1}{\sigma} \text {. }
$$

The quantity

$$
\sigma_{c}=1 / \mathcal{P}_{0},
$$


is the reinfection threshold. When $\sigma>\sigma_{c}$ the disease may spread and persist inside the community where the individuals live. Note that in classical SIR models the presence of an endemic state is due to the replenishment of susceptibles ensured by demography [20], which is not the case here.

The local stability analysis of $E_{1}$ requires the Jacobian matrix of system (10):

$$
J=\left[\begin{array}{cccc}
J_{11} & -\beta I+\sigma \beta I & -\beta I+\psi \beta I & 0 \\
-\sigma \beta R+\gamma & -\sigma \beta I & 0 & 0 \\
-\varphi(M)-\psi \beta V & -\varphi(M) & -\varphi(M)-\psi \beta I & -\varphi^{\prime}(M)(1-I-R-V) \\
a k & 0 & 0 & -a
\end{array}\right]
$$

where

$$
J_{11}=-\beta I+\beta(1-I-R-V)+\sigma \beta R+\psi \beta V-\gamma
$$

Taking into account of (13), (14) and that $V_{1}=0$, it follows

$$
J\left(E_{1}\right)=\left[\begin{array}{cccc}
-\beta I_{1} & -\beta I_{1}+\sigma \beta I_{1} & -\beta I_{1}+\psi \beta I_{1} & 0 \\
0 & -\sigma \beta I_{1} & 0 & 0 \\
-\varphi\left(M_{1}\right) & -\varphi\left(M_{1}\right) & -\varphi\left(M_{1}\right)-\psi \beta I_{1} & 0 \\
a k & 0 & 0 & -a
\end{array}\right]
$$

The eigenvalues are:

$$
\lambda_{1}=-a ; \quad \lambda_{2}=-\sigma \beta I_{1}
$$

and the eigenvalues of the submatrix:

$$
\tilde{J}=\left[\begin{array}{cc}
-\beta I_{1} & -\beta I_{1}+\psi \beta I_{1} \\
-\varphi\left(M_{1}\right) & -\varphi\left(M_{1}\right)-\psi \beta I_{1}
\end{array}\right]
$$

The trace is negative and the determinant is

$$
\operatorname{det} \tilde{J}=\psi \beta^{2} I_{1}^{2}+\psi \beta \varphi\left(M_{1}\right) I_{1}>0
$$

so that $E_{1}$ is locally asymptotically stable.

Remark 1 We remark that:

(i) the stable endemic state $E_{1}$ can be realized thanks to the imperfection of the vaccine, in the sense that when $\psi=0$ in (9) the variable $V$ is always increasing.

(ii) the information index, in the form described in Sect. 3, may be responsible of the onset of sustained oscillations in epidemic models both in the case of delayed information (see e.g. $[12,14,16,17]$ ) and instantaneous information (as it happens when the latency time is included in the model [7]). In all these mentioned cases, the epidemic spread is considered on a long time-scale and demography is taken into account. The analysis in this section clearly shows that sustained oscillations are not possible for the short time-scale SIRI model with information. 

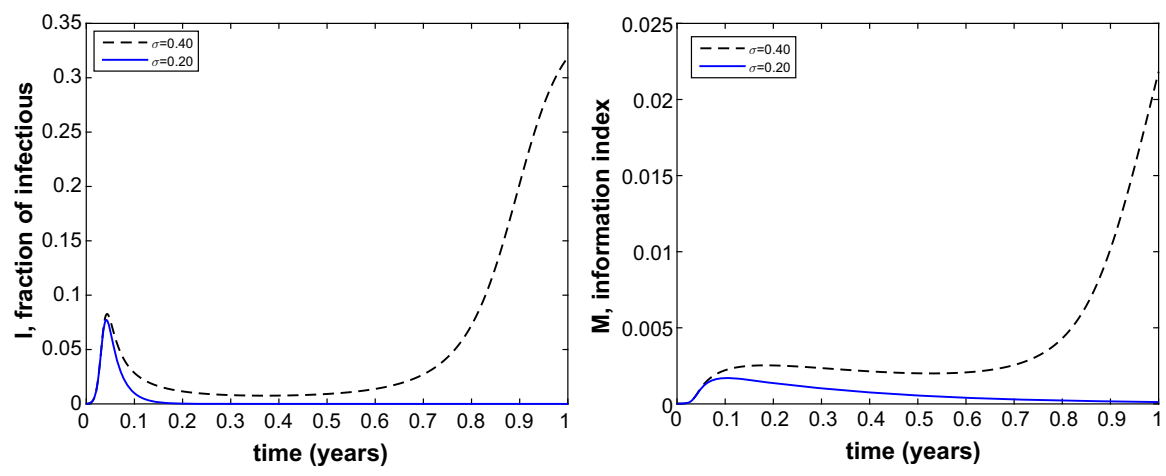

Fig. 2 Time profiles of fraction of infectious $I$ (Left panel) and and information index $M$ (Right panel). The dotted line correspond to a value of the reinfection rate $\sigma$ above the threshold $1 / \mathcal{P}_{0}=0.259$. Therefore the disease is sustained endemically by reinfection. The continuous line correspond to a value of $\sigma$ below the threshold $1 / \mathcal{P}_{0}=0.259$, so that the epidemic will eventually die out. The information coverage is $k=0.2$ and the average time delay of information is $T=120$ days. The other parameter values are given in Table 1

\section{Numerical investigations}

We use epidemiological parameter values based on early estimation of novel coronavirus COVID-19 provided in [28]. The estimation, based on the use of a SEIR metapopulation model of infection within Chinese cities, revealed that the transmission rate within the city of Wuhan, the epicenter of the outbreak, was $1.07 \mathrm{day}^{-1}$, and the infectious period was 3.6 days (so that $\gamma=0.27 \mathrm{day}^{-1}$ ).

Therefore the BRN given in (11) is $\mathcal{P}_{0}=3.85$ (of course, in agreement with the estimate in [28]), and the value $\sigma_{c}:=1 / \mathcal{P}_{0}=0.259$ is the threshold for the infection rate.

For vaccinated individuals, the relative susceptibility (compared to an unvaccinated individuals) is set $\psi=0.15$, which means that vaccine administration reduces the transmission rate by $85 \%$ (vaccine efficacy $=0.85$ ). This value falls within the estimates for the most common vaccine used in the USA, where vaccine efficacy ranges between 0.75 and 0.95 (see Table 9.3, p. 222, in [24]).

As for the relative susceptibility of recovered individuals, we consider two relevant baseline cases:

(i) Case I: $\sigma=0.2$. This value is representative of a reinfection value below the reinfection threshold $\sigma_{c}$;

(i) Case II: $\sigma=0.4$. This value is representative of a reinfection value above the reinfection threshold $\sigma_{c}$.

The information parameter values are mainly guessed or taken from papers where the information dependent vaccination is used [5,14]. The information coverage $k$ ranges from a minimum of 0.2 (i.e. the public is aware of $20 \%$ of the prevalence) to 1 . The average time delay of information ranges from the hypothetical case of immediate information $(T=0)$ to a delay of 120 days. 
Fig. 3 Time profile of incidence. The reinfection rate $\sigma$ above the threshold $1 / \mathcal{P}_{0}=0.259$. The dotted line correspond to a low value of the information coverage $(k=0.2)$. A greater value ( $k=0.4$, continuous line) results in the elimination of the disease. The average time delay of information is $T=120$ days. The other parameter values are given in Table 1

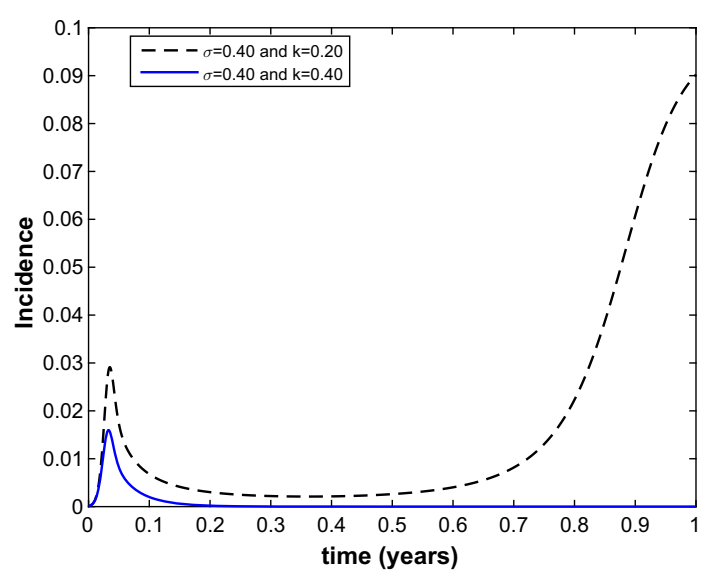

The description and baseline values of the parameters are presented in Table 1. The initial data reflect a scenario in which a small portion of infectious is present in the community at time of administrating the vaccine. Furthermore, coherently with the initial data mentioned in Sect. 4, we assume that:

$$
I(0)=10^{-4}, \quad R(0)=0, \quad V(0)=0, \quad M(0)=k I(0),
$$

and, clearly, $S(0)=1-I(0)$.

According to the analysis made in Sect. 4, values of $\sigma$ below the threshold $\sigma_{c}$ implies that the epidemic will eventually die out. When $\sigma$ is above $\sigma_{c}$, then the disease is sustained endemically by reinfection. This behavior is illustrated in Fig. 2, where it is considered the worst possible scenario, where $k=0.2$ and $T=120$ days.

In Fig. 2, left panel, the continuous line is obtained for $\sigma=0.2$. Vaccination is not able to influence the outbreak, due to the large delay. However, even though an epidemic peak occurs after three weeks, thereafter the disease dies out due to the low level of reinfection. The case $\sigma=0.4$ is represented by the dotted line. As expected, the reinfection is able to 'restart' the epidemic. The trend (here captured for one year) would be to asymptotically converge to the endemic equilibrium $E_{1}$.

The corresponding time evolution of the information index $M$ is shown in Fig. 2, right panel. In particular, in the elimination case $(\sigma=0.2)$, the information index reaches a maximum of 0.002 (approx.) which corresponds to a vaccination rate of $51.5 \%$ (see Fig. 1). After that, it declines but, due to memory of past events, the information index is still positive months after the elimination of the disease. The 'social alarm' produced in the case $\sigma=0.4$ is somehow represented by the increasing continuous curve in Fig. 2, right panel. At the end of the time frame it is $M \approx 0.022$ which corresponds to a vaccination rate of $91 \%$.

In summary, a large reinfection rate may produce a large epidemic. However, even in this worst scenario, the feedback produced by behavioral changes due to information may largely affect the outbreak evolution. In Fig. 3 we see the effect of an higher information coverage $(k=0.4$, left panel) on the incidence of the disease which, for 

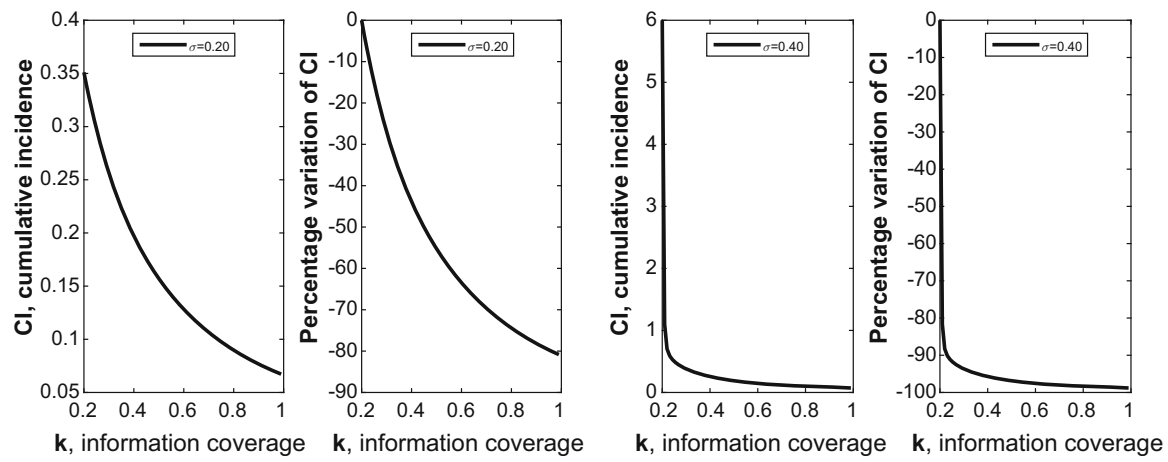

Fig. 4 Cumulative incidence of the disease (first and third panel from the left) and percentage variation of the cumulative incidence (second and fourth panel) by varying the information coverage $k$. The first two panels refer to the case $\sigma=0.2$. The third and fourth panel refer to the case $\sigma=0.4$. The parameter values are given in Table 1

model (10), is given by the quantity:

$$
\text { incidence }(t)=\beta S(t) I(t)+\sigma \beta R(t) I(t)+\psi \beta V(t) I(t) \text {. }
$$

More informed people react and vaccinate and this, in turn, contribute to the elimination of the disease. Therefore, a threshold value $k_{c}$ exists above which the disease can be eliminated. An insight on the overall effect of parameter $k$ on the epidemic may be determined by evaluating how it affects the cumulative incidence (CI),

$$
\mathrm{CI}=\int_{0}^{t_{f}}(\beta S I+\sigma \beta R I+\psi \beta V I) d t
$$

i.e. total number of new cases in the time frame $\left[0, t_{f}\right]$. We also introduce the following index

$$
\mathrm{RCCI}, p:=\frac{\mathrm{CI}\left(p_{1}\right)-\mathrm{CI}\left(p_{2}\right)}{\mathrm{CI}\left(p_{2}\right)}
$$

which measures the relative change of cumulative incidence for two different values, say $p_{1}$ and $p_{2}$, of a given parameter $p$ over the simulated time frame (in other words, the percentage variation of the cumulative incidence varying $p$ from $p_{2}$ ).

In Fig. 4 (first plot from the left) it is shown the case of a reinfection value $\sigma=0.2$, that is under the reinfection threshold. It can be seen how CI is declining with increasing $k$. In Fig. 4 (second plot from the left) a comparison with the case of low information coverage, $k=0.2$, is given: a reduction till $80 \%$ of CI may be reached by increasing the value of $k$ till $k=0.99$. When the reinfection value is $\sigma=0.4$ (Fig. 4 , third and fourth plot), that is above the reinfection threshold, the 'catastrofic case' is represented in correspondence of $k=0.2$. This case is quickly recovered by increasing $k$, as we already know from Fig. 3, because of the threshold value $k_{c}$, between 0.2 and 0.3 , which allows to pass from the endemic to no-endemic asymptotic state. Then, again 

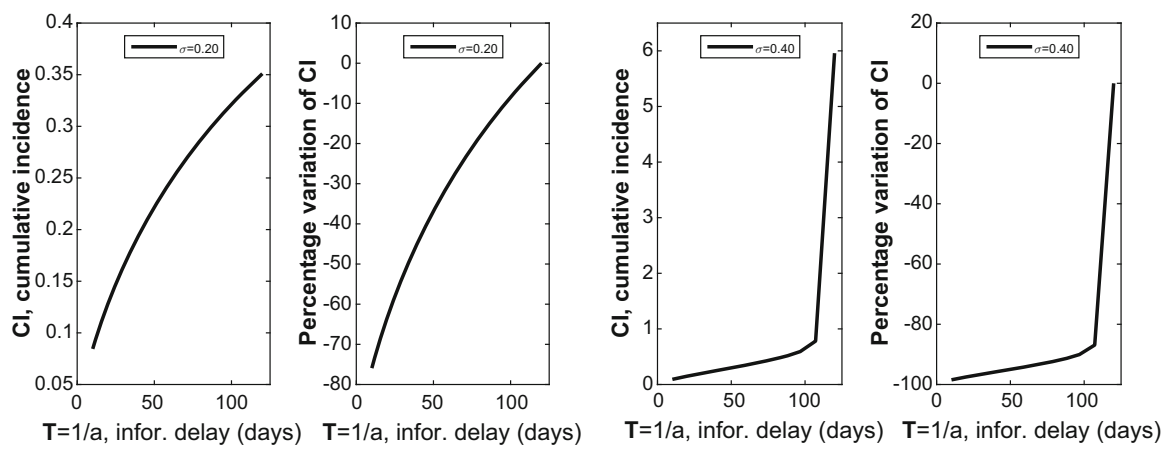

Fig. 5 Cumulative incidence of the disease (first and third panel from the left) and percentage variation of the cumulative incidence ( two panels refer to the case $\sigma=0.2$. The third and fourth panel refer to the case $\sigma=0.4$. The parameter values are given in Table 1

$\mathrm{CI}$ is declining with increasing $k$. This means that when reinfection is high, the effect of information coverage is even more important. In fact, in this case the prevalence is high and a high value of $k$ result in a greater behavioral response by the population.

In Fig. 5 it is shown the influence of the information delay $T$ on CI. In the case $\sigma=0.2$ CI grows concavely with $T$ (first plot from the left). In Fig. 5 (second plot) a comparison with the case of maximum information delay, $T=120$ days, is given: a reduction till $75 \%$ of CI may be reached by reducing the value of $T$ till to very few days. When the reinfection value is $\sigma=0.4$ (Fig. 5, third and fourth plot), that is above the reinfection threshold, CI increases convexly with $T$. A stronger decreasing effect on CI can be seen by reducing the delay from $T=120$ days to $T \approx 90$, and a reduction till $98 \%$ of CI may be reached by reducing the value of $T$ till to very few days.

\section{Conclusions}

In this paper we have investigated how a hypothetical vaccine could affect a coronavirus epidemic, taking into account of the behavioral changes of individuals in response to the information about the disease prevalence.

We have first considered a basic SIRI model. Such a model contains the specific feature of reinfection, which is typical of coronaviruses. Reinfection may allow the disease to persist even when the time-scale of the outbreak is small enough to neglect the demography (births and natural death).

Then, we have implemented the SIRI model to take into account of: (i) an available vaccine to be administrated on voluntary basis to susceptibles; (ii) the change in the behavioral vaccination in response to information on the status of the disease.

We have seen that the disease burden, expressed through the cumulative incidence, may be significantly reduced when the information coverage is high enough and/or the information delay is short. When the reinfection rate is above the critical value, a relevant role is played by recovered individuals. This compartment offers a reservoir 
of susceptibles (although with a reduced level of susceptibility) and if not vaccinate may contribute to the re-emergence of the disease. On the other hand, in this case a correct and quick information may play an even more important role since the social alarm produced by high level of prevalence results, in turn, in high level of vaccination rate and eventually in the reduction or elimination of the disease.

The model on which this investigation is based is intriguing since partial immunity coupled to short-time epidemic behavior may lead to not trivial epidemic dynamics (see the 'delayed epidemic' case, where an epidemics initially may decrease to take off later [27]). However, it has many limitations in representing the COVID-19 propagation. For example, the model represents the epidemics in a closed community over a relatively short time-interval and therefore it is unable to capture the complexity of global mobility, which is one of the main concerns related to COVID-19 propagation. Another limitation, which is again related to the global aspects of epidemics like SARS and COVID-19, is that we assume that individuals are influenced by information on the status of the prevalence within the community where they live (i.e. the fraction $I$ is part of the total population) whereas local communities may be strongly influenced also by information regarding far away communities, which are perceived as potential threats because of global mobility.

Moreover, in absence of treatment and vaccine, local authorities face with coronavirus outbreak using social distancing measures, that are not considered here: individuals are forced to be quarantined or hospitalized. Nevertheless, contact pattern may be reduced also as response to information on the status of the disease. In this case the model could be modified to include an information-dependent contact rate, as in [5,7]. Finally, the model does not include the latency time and the diseaseinduced mortality is also neglected (at the moment, the estimate for COVID-19 is at around $2 \%$ ). These aspects will be part of future investigations.

Acknowledgements This work has been performed under the auspices of the Italian National Group for the Mathematical Physics (GNFM) of National Institute for Advanced Mathematics (INdAM).

\section{Compliance with ethical standards}

Conflict of interest The author states that there is no conflict of interest.

\section{References}

1. Agenzia Nazionale Stampa Associata: Mascherine sold out in farmacie Roma. http://www.ansa. it/canale_saluteebenessere/notizie/sanita/2020/01/27/virus-cina-mascherine-sold-out-in-farmacieroma_ce7aaede-368c-41a1-9f84-e77177058a27.html. Accessed 30 Jan 2020 (in Italian)

2. Anastassopoulou, C., Russo, L., Tsakris, A., Siettos, C.: Data-based analysis, modelling and forecasting of the novel coronavirus (2019-nCoV) outbreak. medRxiv preprint https://www.medrxiv.org/content/ 10.1101/2020.02.11.20022186v1.full.pdf. Accessed 21 Feb 2020

3. Anderson, R.M., May, R.M.: Infectious Diseases of Humans. Dynamics and Control. Oxford University Press, Oxford (1991)

4. Brauer, F., van den Driessche, P., Wu, J.: Mathematical Epidemiology. Lecture Notes in Mathematics. Mathematical Biosciences Subseries, vol. 1945. Springer, Berlin (2008)

5. Buonomo, B., Della Marca, R.: Oscillations and hysteresis in an epidemic model with informationdependent imperfect vaccination. Math. Comput. Simul. 162, 97-114 (2019) 
6. Buonomo, B., d'Onofrio, A., Lacitignola, D.: Global stability of an SIR epidemic model with information dependent vaccination. Math. Biosci. 216, 9-16 (2008)

7. Buonomo, B., d'Onofrio, A., Lacitignola, D.: Globally stable endemicity for infectious diseases with information-related changes in contact patterns. Appl. Math. Lett. 25, 1056-1060 (2012)

8. Buonomo, B., d'Onofrio, A., Lacitignola, D.: Modeling of pseudo-rational exemption to vaccination for SEIR diseases. J. Math. Anal. Appl. 404, 385-398 (2013)

9. Callow, K.A., Parry, H.F., Sergeant, M., Tyrrell, D.A.J.: The time course of the immune response to experimental coronavirus infection of man. Epidemiol. Infect. 105, 435-446 (1990)

10. Capasso, V.: Mathematical Structures of Epidemic Systems. Springer, Berlin (1993)

11. Danchin, A., Ng, T.W.P., Turinici, G.: A new transmission route for the propagation of the SARSCoV-2 coronavirus. medRxiv preprint https://doi.org/10.1101/2020.02.14.20022939v1. Accessed 21 Feb 2020

12. d'Onofrio, A., Manfredi, P.: Information-related changes in contact patterns may trigger oscillations in the endemic prevalence of infectious diseases. J. Theor. Biol. 256, 473-478 (2009)

13. d'Onofrio, A., Manfredi, P.: Bistable endemic states in a susceptible-infectious-susceptible model with behavior-dependent vaccination. In: Chowell, G., Hyman, J. (eds.) Mathematical and Statistical Modeling for Emerging and Re-emerging Infectious Diseases, pp. 341-354. Springer, New York (2016)

14. d'Onofrio, A., Manfredi, P., Salinelli, E.: Vaccinating behaviour, information, and the dynamics of SIR vaccine preventable diseases. Theor. Popul. Biol. 71, 301-317 (2007)

15. d'Onofrio, A., Manfredi, P., Salinelli, E.: Bifurcation thresholds in an SIR model with informationdependent vaccination. Math. Model. Nat. Phenom. 2, 26-43 (2007)

16. d'Onofrio, A., Manfredi, P., Salinelli, E.: Fatal SIR diseases and rational exemption to vaccination. Math. Med. Biol. 25, 337-357 (2008)

17. d'Onofrio, A., Manfredi, P., Poletti, P.: The impact of vaccine side effects on the natural history of immunization programmes: an imitation-game approach. J. Theor. Biol. 273, 63-71 (2011)

18. Gomes, M.G.M., White, L.J., Medley, G.F.: Infection, reinfection, and vaccination under suboptimal immune protection: epidemiological perspectives. J. Theor. Biol. 228, 539-549 (2004)

19. Isaacs, D., Flowers, D., Clarke, J.R., Valman, H.B., MacNaughton, M.R.: Epidemiology of coronavirus respiratory infections. Arch. Dis. Child. 58, 500-503 (1983)

20. Katriel, G.: Epidemics with partial immunity to reinfection. Math. Biosci. 228, 153-159 (2010)

21. Keeling, M.J., Rohani, P.: Modeling Infectious Diseases in Humans and Animals. Princeton University Press, Princeton (2008)

22. Kumar, A., Srivastava, P.K., Gupta, R.P.: Nonlinear dynamics of infectious diseases via informationinduced vaccination and saturated treatment. Math. Comput. Simul. 157, 77-99 (2019)

23. Manfredi, P., d'Onofrio, A. (eds.): Modeling the Interplay Between Human Behavior and the Spread of Infectious Diseases. Springer, New York (2013)

24. Martcheva, M.: An Introduction to Mathematical Epidemiology. Texts in Applied Mathematics, vol. 61. Springer, New York (2015)

25. Martins, J., Pinto, A.: Bistability of evolutionary stable vaccination strategies in the reinfection SIRI model. Bull. Math. Biol. 79, 853-883 (2017)

26. MacDonald, N., MacDonald, N.: Biological Delay Systems: Linear Stability Theory, vol. 9. Cambridge University Press, Cambridge (2008)

27. Nakata, Y., Omori, R.: The change of susceptibility following infection can induce failure to predict outbreak potential for $R_{0}$. Math. Biosci. Eng. 16, 813-830 (2019)

28. Read, J.M., Bridgen, J.R.E., Cummings, D.A.T., Ho A., Jewell, C.P.: Novel coronavirus 2019-nCoV: early estimation of epidemiological parameters and epidemic predictions. medRxiv preprint https:// www.medrxiv.org/content/10.1101/2020.01.23.20018549v1.full.pdf. Accessed 31 Jan 2020

29. World Health Organization: Cumulative Number of Reported Probable Cases of SARS. https://www. who.int/csr/sars/country/2003_07_11/en/. Accessed 30 Jan 2020

30. World Health Organization: Middle East Respiratory Syndrome Coronavirus (MERS-CoV). https:// www.who.int/emergencies/mers-cov/en/. Accessed 30 Jan 2020

31. World Health Organization: Novel Coronavirus (2019-nCoV). https://www.who.int/emergencies/ diseases/novel-coronavirus-2019/. Accessed 30 Jan 2020

32. World Health Organization: Director-General's remarks at the media briefing on 2019-nCoV on 11 February 2020. https://www.who.int/dg/speeches/detail/who-director-general-s-remarks-at-themedia-briefing-on-2019-ncov-on-11-february-2020. Accessed 12 Feb 2020 
33. Wang, Z., Bauch, C.T., Bhattacharyya, S., d'Onofrio, A., Manfredi, P., Perc, M., Perra, N., Salathe, M., Zhao, D.: Statistical physics of vaccination. Phys. Rep. 664, 1-113 (2016)

34. Wu, L.P., Wang, N.C., Chang, Y.H., Tian, X.Y., Na, D.Y., Zhang, L.Y., Zheng, L., Lan, T., Wang, L.F., Liang, G.D.: Duration of antibody responses after severe acute respiratory syndrome. Emerg. Infect. Dis. 13(10), 1562 (2007)

35. Zhu, L., Zhou, X., Li, Y., Zhu, Y.: Stability and bifurcation analysis on a delayed epidemic model with information-dependent vaccination. Physica Scripta 94, 125202 (2019)

Publisher's Note Springer Nature remains neutral with regard to jurisdictional claims in published maps and institutional affiliations. 